\title{
LOCALIZED SOLUTIONS OF NONLINEAR WAVE EQUATIONS
}

\author{
BY ERIC H. ROFFMAN ${ }^{1}$
}

Communicated by M. H. Protter, June 2, 1969

We consider complex valued solutions $\phi$ of nonlinear wave equations of the form

$$
\square \phi=\phi_{t t}-\Delta \phi=-\phi v\left(|\phi|^{2}\right)
$$

where $v$ is the derivative of a positive definite potential $V$. That is

(2) $\quad \frac{d V(a)}{d a}=v(a)$ and $\quad V(a) \geqq 0 \quad$ with $V(a)=0 \quad$ iff $a=0$.

We suppose $v(0)=m^{2}>0$.

A solution $\phi$ with finite energy is called localized if there is an $\epsilon>0$ such that

$$
\sup _{x}|\phi(x, t)|=M(t)>\epsilon
$$

whenever $\phi$ exists.

Theorem. If, for some $a_{0}$

$$
V\left(a_{0}\right)<m^{2} a_{0}
$$

then equation (1) has localized solutions.

The proof is based on the conservation of energy $\&$ and charge $\mathcal{Q}$

$$
\begin{aligned}
& \mathcal{E}=\int\left\{\left|\phi_{t}\right|^{2}+\sum_{i=1}^{N}\left|\phi_{x_{i}}\right|^{2}+V\left(|\phi|^{2}\right)\right\} d x, \\
& Q=\operatorname{Im} \int\left(\phi_{t} \bar{\phi}\right) d x .
\end{aligned}
$$

Suppose that $|\phi|^{2}<\epsilon$. Then, from (2)

$$
V\left(|\phi|^{2}\right)>\left(m^{2}-\delta\right)|\phi|^{2}
$$

where $\delta$ tends to zero if $\epsilon$ does.

By the Schwartz inequality and (7) we easily deduce that

'Supported in part by AEC Contract number AT(30-1) 3668-B.

I would like to thank Professor P. D. Lax for help in preparing this article for publication. 


$$
\mathcal{Q} \leqq \frac{1}{2 \sqrt{m^{2}-\delta}} \mathcal{E}
$$

However, consider now the initial data

$$
\phi(x, 0)=a_{0} \eta(x), \quad \phi_{t}(x, 0)=i m a_{0} \eta(x),
$$

where

$$
\begin{aligned}
\eta(x) & =1 \quad \text { for }|x|<R, \\
& =R+1-|x| \quad \text { for } R \leqq x \leqq R+1, \\
& =0 \quad \text { for } R+1 \leqq|x| .
\end{aligned}
$$

It is easy to see that if (4) holds, then for large enough $R$

$$
Q>\frac{1}{2 \sqrt{m^{2}-\delta}} \varepsilon \text {. }
$$

Since $Q$ and $\varepsilon$ are independent of time, it then follows that $M(t)>\epsilon>0$ whenever $\phi$ exists. This shows that $\phi$ with initial data (9) is localized.

Next, we point out that for some equations of the type (1), there is a particularly interesting family of localized solutions.

Consider a function of the form

$$
\phi_{\nu, \alpha, a}(x, t)=e^{i \nu(\alpha t-\beta x)} \eta(\alpha x-\beta t+a) .
$$

This is a solution of equation (1) if $\alpha^{2}-\beta^{2}=1, \nu^{2}<m^{2}$ and if $\eta$ satisfies

$$
\Delta \eta=\eta v\left(|\eta|^{2}\right)-\nu^{2} \eta
$$

with $\eta$ vanishing exponentially as $|x| \rightarrow \infty$.

I have shown, by numerical integration of equations (5), (6), and (12), that there exist potentials $V$ (in one-, two-, and three-dimensions) for which solutions $\phi$ of the form (11) exist and satisfy (10).

Consider now the initial conditions

$$
\Phi=\sum_{i} \phi_{\nu_{i} \alpha_{i} a_{i}}\left(x, t_{0}\right), \quad \Phi_{t}=\sum_{i} \frac{d}{d t} \phi_{v_{i} \alpha_{i} a_{i}}\left(x, t_{0}\right) .
$$

If each $\phi_{v_{i} \alpha_{i} a_{i}}(x, t)$ satisfies (10), then it is easy to see that if each $\left|a_{i}-a_{j}\right|=R_{i j}$ is sufficiently large, the initial data (13) also satisfy (10), and so the solution $\Phi(x, t)$ with these initial data is localized.

State University of New York, Stony Brook, New York 11790 